\title{
Prolonged positive airway pressure for severe neonatal tracheobronchomalacia
}

\author{
B L PIZER, A P FREELAND, AND A R WILKINSON \\ Neonatal Unit and Department of Otolaryngology, John Radcliffe Hospital, Oxford
}

\begin{abstract}
SUMMARY A very low birthweight preterm baby with respiratory distress at birth was found to have severe congenital tracheobronchomalacia. Continuous positive airway pressure was given through an endotracheal tube without tracheostomy for 15 weeks before unassisted respiration could be maintained. Diagnosis was made and progress monitored by laryngobronchoscopy on three occasions.
\end{abstract}

Disorders of the large airways in the newborn are uncommon and the signs may be attributed to pulmonary disease, particularly in preterm babies. This case of a baby with severe congenital tracheobronchomalacia illustrates the difficulties in making the diagnosis in a very low birthweight infant and the long term support of ventilation necessary for a successful outcome.

\section{Case report}

A boy was born by emergency caesarean section weighing $1060 \mathrm{~g}$ after prolapse of the umbilical cord at 27 weeks' gestation. The parents were white and unrelated. The pregnancy had been otherwise normal.

At birth he cried and established spontaneous regular breathing but before 1 minute of age developed intercostal and sternal recession and became cyanosed. He was resuscitated with endotracheal intubation and positive pressure ventilation with rapid improvement. Artificial ventilation was continued until admission to the intensive care nursery when his temperature was $36.0^{\circ} \mathrm{C}$, blood glucose concentration $5.0 \mathrm{mmol} / 1(90 \mathrm{mg} / 100 \mathrm{ml})$, and packed cell volume $40 \%$. Signs of respiratory distress persisted. Examination showed a normal preterm male infant without any obvious malformations or dysmorphic features. An umbilical arterial catheter was inserted, a full infection screen carried out, and treatment with antibiotics started. Chest radiographs showed no signs of hyaline membrane disease or other pulmonary disease. The lecithin: sphingomyelin ratio measured in pharyngeal secretions aspirated at 2 hours of age was 3.6 (normal $>1.8$ ). Artificial ventilation could be rapidly de- creased to six breaths per minute with a fractional inspiratory oxygen of $0 \cdot 23$. Initial bacterial cultures were negative. Continuous positive airway pressure was then applied but an attack of apnoea occurred and treatment with aminophylline was begun on the fourth day. On the seventh day a symptomatic patent ductus arteriosus was diagnosed and assisted ventilation increased. Despite restriction in fluid intake and treatment with indomethacin, the signs persisted and on the 18th day ligation of the ductus arteriosus was performed. Postoperative recovery was complicated by pulmonary collapse and consolidation. On day 21 he was weaned to continuous positive airway pressure of $5 \mathrm{~cm} \mathrm{H}_{2} \mathrm{O}$.

Between day 21 and 31 extubation was attempted four times but on each occasion was unsuccessful. Stridor was followed by attacks of apnoea, episodes of bradycardia, and cyanosis and chest radiographs showed bilateral upper lobe consolidation. Bronchoscopy showed no vocal cord palsy or subglottic stenosis, but there was pronounced tracheal collapse on withdrawal of the bronchoscope, establishing a diagnosis of tracheomalacia. He remained stable while receiving continuous positive airway pressure for the next 10 days. At 6 weeks of age upper lobe collapse, fluctuating between the right and left lung, suggested bronchomalacia. On day 47 the endotracheal tube was removed twice, but reintubation became necessary after four and six hours, respectively.

Repeat bronchoscopy on day 58 again showed normal vocal cords without subglottic stenosis but pronounced collapse of the posterior wall of the trachea on expiration. Management continued with continuous positive airway pressure. From day 79 a regimen of alternating continuous positive airway pressure and spontaneous breathing through the endotracheal tube of humidified air/oxygen was begun. Initially, spontaneous breathing was allowed for five minutes each hour increasing to 20 minutes each hour. On day 86 after collapse of the right lung a third bronchoscopy was carried out. Secretions were removed and signs of tracheomalacia were still present. On day 108, at a postconceptional age of 42 weeks, he was extubated. This was followed by considerable inspiratory stridor and recession but no attacks of apnoea, and blood gases remained within 
the normal range. Stridor persisted, increasing when he was awake or agitated, but gradually diminished in intensity over the next month, and the fractional inspiratory oxygen was reduced from $0 \cdot 35$ to air. Upper lobe collapse and consolidation improved. Throughout the first 15 weeks he thrived on enteral milk feeds of his mother's expressed breast milk. Bottle feeding was successfully introduced on day 114.

He was discharged aged 5 months. He continued to have intermittent stridor but otherwise remained well and thrived. There were no abnormal neurological signs and cerebral ultrasound scans yielded normal results. At 18 months his stridor, though still present, is improving. He has had no appreciable infections and no hospital admissions and his development is normal.

\section{Discussion}

Tracheomalacia, which may be congenital or acquired, is defined as weakness of the tracheal wall from softening of the supporting cartilages with hypotonia of the myoelastic elements and may involve the whole of the trachea or occur as a localised phenomenon. ${ }^{1}$ Only a few cases of acquired tracheomalacia have been reported in children less than 1 year of age and none in extremely low birthweight preterm infants. It may follow prolonged intubation, especially with a shouldered endotracheal tube, chest injury, external vascular compression, or tracheo-oesophageal fistula. In a series of 127 cases diagnosed by bronchoscopy 42 $(32 \%)$ had major associated abnormalities and 55 $(43 \%)$ had unilateral or bilateral bronchomalacia. ${ }^{2}$ The commonest presenting signs were stridor or wheezy breathing, recurrent bronchitis, chronic cough, and cyanosis. Although acquired tracheomalacia cannot be excluded in the patient described, it seems unlikely as signs were of early onset and bronchomalacia was also present.

Congenital tracheomalacia exists in two distinct forms, a common mild variant and an uncommon severe form. The mild variant may be misdiagnosed as wheezy bronchitis or asthma in infants below 1 year of age. It represents an exaggeration of the normal softness and pliability of the tracheal supportive structures. A series of 15 cases showed that symptoms started from 5 days to 4 months and resolved with conservative management, without mortality, between 3 and 18 months. ${ }^{3} \mathrm{~A}$ report of two term infants with severe bronchomalacia described successful management with $5 \mathrm{~cm} \mathrm{H}_{2} \mathrm{O}$ continuous positive airway pressure for 13 and 14 weeks, respectively. ${ }^{4} \mathrm{~A}$ child with Larsen's syndrome has been reported in whom continuous positive airway pressure, given for 22 months, improved pulmonary mechanics. ${ }^{5}$

In four cases of severe tracheomalacia, diagnosed by broncoscopy, tracheostomy was performed between 1 week and 21 months of age. ${ }^{6}$ Two had associated bronchomalacia and one died from an associated viral infection. A pronounced worsening of the symptoms with crying or agitation and upper respiratory tract infections was noted. The diagnosis of severe congenital tracheomalacia has been made by fluoroscopy or lateral inspiratory and expiratory radiographs. The latter may, however, be normal. ${ }^{6}$ In the intensive care nursery bronchoscopy with a rigid ventilating bronchoscope will allow visualisation of the larynx, trachea, and major bronchi and enable aspiration of secretions. Intrinsic or intraluminal lesions can be excluded and the diagnosis made safely. Treatment with continuous positive airway pressure prevents bronchial collapse and in conjunction with physiotherapy may be required for an extended period until the bronchi become more rigid.

\section{References}

${ }^{1}$ Baxter JD, Dunbar JS. Tracheomalacia. Ann Otol Rhinol Laryngol 1963;72:1013.

2 Couvreur J, Grimfeld A, Tournier G, et al. La dyskinesie tracheale (tracheomalacie) chez l'enfant. Reflexions à propos de 127 cas reconnus par endoscopie. Ann Pediatr (Paris) 1980;27:561-70.

${ }^{3}$ Levin SJ, Adler P, Scherer RA. Collapsible trachea (tracheomalacia). A non-allergic cause of wheezing in infancy. Ann Allergy 1965;22:22-5.

${ }^{4}$ Neijens HJ, Kerrebijn KF, Smalhout B. Successful treatment with CPAP of two infants with bronchomalacia. Acta Paediatr Scand 1978;67:293-6.

5 Kanter RK, Pollack MM, Wright WW, Grundfast KM. Treatment of severe tracheobronchomalacia with continuous positive airway pressure (CPAP). Anesthesiology 1982;57:54-6.

${ }^{6}$ Cogbill TH, Moore FA, Accurso FJ, Lilly JR. Primary tracheomalacia. Ann Thorac Surg 1983;35(5):538-41.

Correspondence to Dr Andrew Wilkinson, Neonatal Unit, John Radcliffe Maternity Hospital, Headington, Oxford OX3 9DU, England.

Received 12 April 1986 